\title{
Ultrasound aspect of mesenteric panniculitis. Case report.
}

\author{
Radu Badea1, Liliana Chiorean², Dana Damian³, Geza Molnar ${ }^{4}$, Ofelia Anton²
}

${ }^{1}$ Department of Ultrasonography, ${ }^{2}$ Department of Radiology and Computed Tomography, ${ }^{3}$ Department of Gastroenterology, ${ }^{4}$ Department of Surgery, "Octavian Fodor" Institute of Gastroenterology and Hepatology, "Iuliu Haţieganu" University of Medicine and Pharmacy, Cluj-Napoca, Romania

\begin{abstract}
Mesenteric panniculitis represents a cronic inflammation of the adipose tissue of the mesentery which can present sometimes with the imagistic aspect of one or multiples pseudotumors. We present the case of female patient in which mesenteric panniculitis was found accidentally during a routine abdominal ultrasound examination leading to differential diagnostics problems. Color Doppler and contrast enhancement ultrasonography were helpful for diagnosis evidencing a benign circulatory signal.
\end{abstract}

Keywords: mesenteric panniculitis, contrast enhancement ultrasonography

\section{Introduction}

Mesenteric panniculitis is a chronic, unspecific and idiopathic inflammation of the adipose tissue in the mesentery. It represents a phase of fatty tissue degeneration at this level, starting with lipodystrophy, continuing with inflammation and ending in retraction and fibrosis (commonly named retractile mesenteritis) [1]. Macroscopically it may take three aspects: thickening and diffuse infiltration of the mesentery ( $42 \%$ of cases), solitary mesenteric mass ( $32 \%$ of cases), or multiple mesenteric masses ( $26 \%$ of cases) [2]. Before the "imaging era" mesenteric panniculitis was rarely diagnosed. Nowadays the condition is frequently encountered, usually as a chance finding during examinations for other diseases [3]. Its cause continues to be uncertain, an autoimmune response

Received 12.01.2013 Accepted 25.02.2013

Med Ultrason

2013, Vol. 15, No 3, 247-249

Corresponding author: Badea Radu,

Institute of Gastroenterology and

Hepatology, Department of Ultrasound

"Iuliu Hatieganu" University of Medicine and Pharmacy Cluj Napoca,

19-21 Croitorilor Str,

400163, Cluj Napoca, Romania

Tel: +40740084968 , Fax: +4026453424

Email: rbadea@umfcluj.ro to an unknown source and mesenteric ischemia being suggested as possible etiopathogenetic mechanisms [4].

We present the case of a 70-year-old female patient in which mesenteric panniculitis was found accidentally during a routine abdominal ultrasound (US) examination.

\section{Case presentation}

A a 70-year-old female patient with documented essential arterial hypertension, liver steatosis, high triglycerides levels, hyperplasic polypi in the ascending colon removed endoscopically, was referred for abdominal US evaluation. The patient's main complaint was represented by diffuse, unspecified abdominal pain.

Abdominal US evidenced a hypoechogenic mass of $50 / 40 \mathrm{~mm}$ (fig 1a) in the left hypochondrium, between the intestinal loops, which presented vascular sign at the color Doppler investigation (CDI) (fig 1b). Intravenous contrast (Sonovue, $1.6 \mathrm{ccm} / 10 \mathrm{ccm}$ saline; GE 7 device) was administered in order to gather more precise data on the lesion. Contrast-enhanced ultrasound (CEUS) evidenced rapid, non-homogeneous filling of the mass during the arterial time, without a wash-out of the contrast agent during the venous time (fig 1c). CT scan was performed to complete the diagnosis and to exclude other associated conditions. CT revealed the presence of hypertrophic fatty tissue at the level of the mesentery, of 

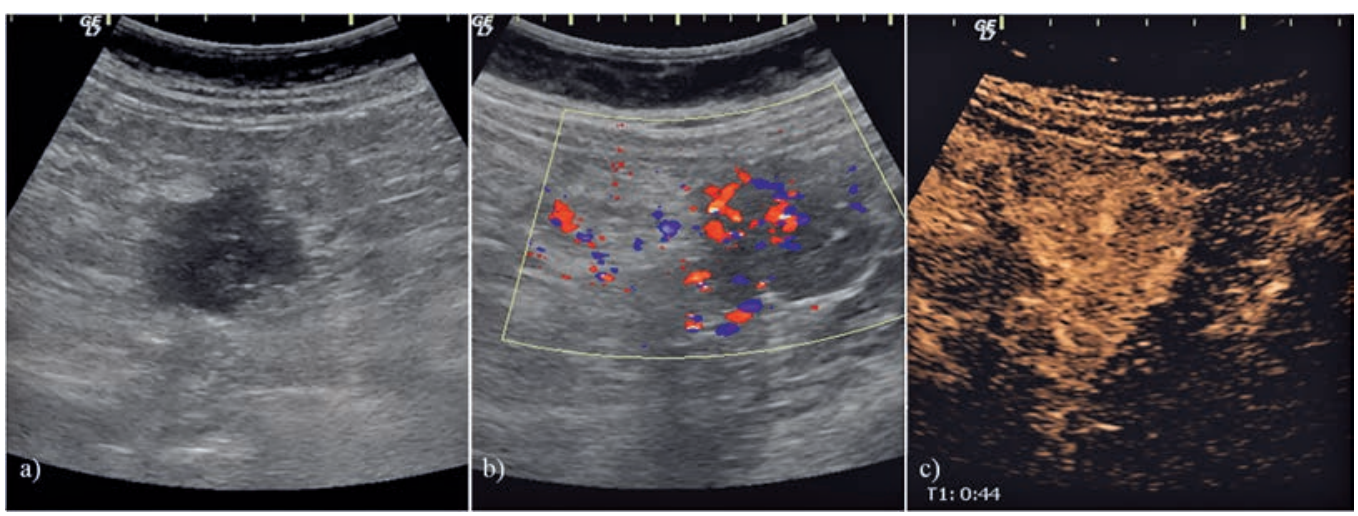

Fig 1. a) Abdominal ultrasonography of the left hypochondrium showing a hypoechoic mass, well delimited, situated between the intestinal loops; b)Doppler examination showing a vascular sign inside the formation; c) CEUS of the tumoral mass (portal venous time, 44 seconds after I.V. injection). Persistent vascular sign may be seen, with branching arrangement of the vessels in space.

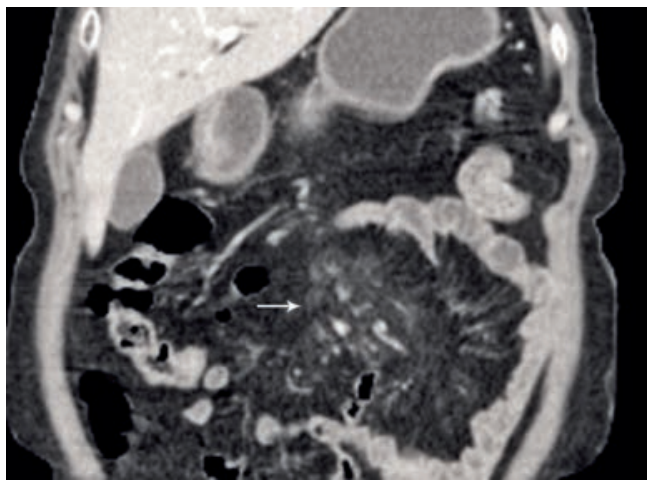

Fig 2. Contrast CT scan (venous time, coronal section). Aspect of "misty mesentery" - increased mesenteric fat density, especially at its root.

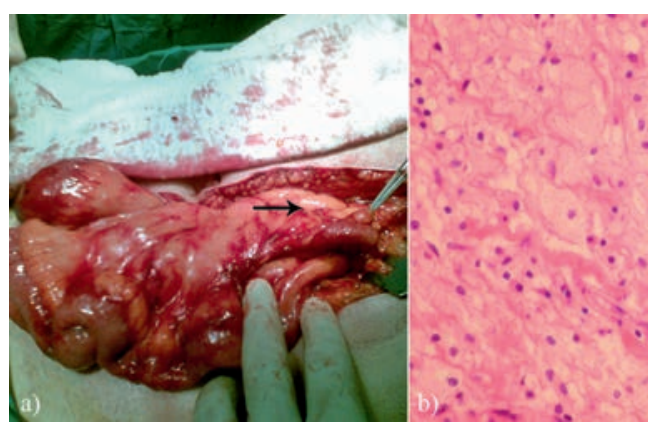

Fig 3. a) Intraoperative aspect of the adipose mesenteric panniculus, hypertrophied, pseudotumoral aspect ; b) Histology of the bioptic sample harvested by operation (hematoxilin-eosin stain, x40 enlargement). Normal lipocytes and blood cells with inflammatory aspect may be seen. slightly higher density, the mesenteric vessels crossing it were surrounded by a less dense peripheral halo (fat ring sign), while the other abdominal organs had normal CT aspect (fig 2). The diagnosis of mesenteric panniculitis was suggested.

Exploratory laparotomy was performed in order to obtain bioptic samples for the pathological examination of the lesion. Several formations were found at the mesenteric level intraoperatively, with diameters between 1-4 cm (fig 3a). Three formations were excised for biopsy. Histopathological examination evidenced lipomatous features, and in section the presence of steato-necrotic areas within the lesions. Microscopic examination (HE stain) evidenced mature adipose tissue lobules with areas of steato-necrosis, infiltrated with numerous xanthomatous cells to which lymphocytes and plasmocytes were associated, disposed mainly around the vessels. In the sections examined two lymph nodes were found. No lipoblasts or nuclear abnormalities were found in the samples examined (fig $3 b$ ). The final diagnosis of mesenteric panniculitis was established. Postoperative evolution was good.

\section{Discussions}

Mesenteric panniculitis is a rare abnormality characterized by the pseudotumoral expansion of mesenteric fat due to variable degrees of steatonecrosis, chronic inflammation and fibrosis [5]. The condition affects mainly men (2-3:1) and is more frequently diagnosed after the age of 50 [6]. The mesentery of the small bowel is mostly affected, mainly located at its root, though the sigmoid mesocolon and the omentum may also be involved [7]. Though in $30-50 \%$ of cases the disease remains asympto- 
matic, patients may present abdominal pain, palpable abdominal masses or intestinal obstruction. The evolution is mostly benign, extremely slow, a process associated with body weight gain. However, in $20 \%$ of the patients mesenteric panniculitis is associated with significant morbidity and debilitating chronic evolution [5]. Before the diagnosis of mesenteric panniculitis is established, pancreatitis and inflammatory bowel diseases should be ruled out [1].

Ultrasonographic modifications in mesenteric panniculitis are usually subtle and often overlooked, the diagnosis being potentially suggested by a change in the echogenicity of mesenteric fat associated with a decrease of mesentery compressibility. These changes are unspecific and may also be found in other diseases involving the mesentery, such as lipomatous tumors. The CDI aspect may evidence small vessels with a linear trajectory and branching spatial arrangement. CEUS evidences the vascularization of the lipomatous mass in the arterial time. During the venous time (between 30 - 120 seconds) the wash-out phenomenon does not occur, which removes the suspicion of malignancy. Examination is not always easy, as the condition occurs mainly in overweight patients.

CT scan is always indicated in the analysis of a mesenteric abnormality evidenced by ultrasound [3]. The CT diagnostic indicator for mesenteric panniculitis is the increased density of the mesenteric fat secondary to inflammation (to about -40 up to $-60 \mathrm{HU}$ ) as compared to the normal values of the retroperitoneal or subcutaneous fat density ( -100 to $-160 \mathrm{HU})$. Sometimes a displacement of the intestinal loops and a mass effect on the neighboring organs is evidenced, as well as the specific peripheral hypo-dense halo around the vessels and lymph nodes in the area. The caliber and trajectory of the vessels are unchanged, while lymph nodules are less than $5 \mathrm{~mm}$ in diameter. The presence of mesenteric lymph nodules larger than $10 \mathrm{~mm}$ is not typical for panniculitis, and fine needle aspiration biopsy should be considered in order to exclude malignancy. The presence of a hyper-dense, discontinuous peripheral ring is also a characteristic feature. The term often used to describe it is "misty mesentery", but it is not specific, as any infiltrative process at the mesenteric level may present this aspect (hemorrhage, edema, lymphoma). It is more frequently located on the left side of the abdomen, along the jejunal mesentery. The typical location, the aspect, the asymptomatic chronic evolution suggest the diagnosis of mesenteric panniculitis in most cases; however, before the diagnosis is established, all other causes of misty mesentery must be carefully ruled out $[8,9]$.

To conclude, mesenteric panniculitis is a benign condition that generally requires minimal treatment. It may be a paraneoplastic condition in some cases, though the association between mesenteric panniculitis and malignant disease is less understood $[5,8]$. The initial diagnosis is imaging, and the final one pathological, based on the evidence of a variable combination of areas of fatty degeneration and necrosis, unspecific inflammatory infiltration, predominantly lymphocytic and fibrosis. Ultrasound examination is decisive in the primary identification of the lesion. CDI and CEUS evidence a circulatory signal, benign in nature. Differential diagnosis must take into account the most frequent mesenteric tumors (lymphoma, lymphosarcoma, desmoid tumors) [10].

\section{References}

1. Buetow MP. Mesenteric panniculitis, Radiology Case of the Month. Applied Radiology 2002; 31: 29-30.

2. Kipfer RE, Moertel CG, Dahlin DC. Mesenteric lipodystrophy. Ann Intern Med 1974; 81: 582-588.

3. van Breda Vriesman AC, Schuttevaer HM, Coerkamp EG et al. Mesenteric panniculitis: US and CT features. Eur Radiol 2004; 14: 2242-2248.

4. Daskalogiannaki M, Voloudaki A, Prassopoulos P et al. CT evaluation of mesenteric panniculitis: prevalence and associated diseases. AJR Am J Roentgenol 2000;174:427-431.

5. Ferrari TC, Couto CM, Vilaça TS et al. An unusual presentation of mesenteric panniculitis. Clinics (Sao Paulo) 2008; 63: 843-844.

6. Parra-Davila E, McKenney MG, Sleeman D et al. Mesenteric panniculitis: case report and literature review. Am Surg 1998; 64: 768-771.

7. Jeong YJ, Kim S, Kwak SW et al. Neoplastic and nonneoplastic conditions of serosal membrane origin: CT findings. Radiographics 2008; 28: 801-817.

8. Pereira JM, Sirlin CB, Pinto PS et al. CT and MR imaging of extrahepatic fatty masses of the abdomen and pelvis: techniques, diagnosis, differential diagnosis, and pitfalls. Radiographics 2005; 25: 69-85.

9. Méndez-Uriburu L, Ahualli J, Méndez-Uriburu J et al. CT appearances of intraabdominal and intrapelvic fatty lesions. AJR Am J Roentgenol 2004; 183: 933-943.

10. Grossman LA, Kaplan HJ, Preuss HJ et al. Mesenteric Panniculitis. JAMA 1963; 183: 318-323 